\title{
A simple and rapid method for the detection and identification of mycobacteria using mycobactin
}

\author{
R. BARCLAY, VANESSA FURST* and I. SMITH*
}

Department of Microbiology, University of Reading, PO Box 228, Reading RG6 2AJ and * School of Pathology, University College and Middlesex School of Medicine, Ridinghouse Street, London W1P 7PN

\begin{abstract}
Summary. A system was developed for the identification of mycobacteria such as Mycobacterium tuberculosis and $M$. avium, by thin layer chromatography of ${ }^{55} \mathrm{Fe}$-labelled mycobactin. Approximately $2 \times 10^{3}$ mycobacteria were detected within $24 \mathrm{~h}$ and little operator time or skill was required. $M$. avium, $M$. intracellulare and $M$. scrofulaceum were found to have lower requirements for iron than other mycobacteria and this may influence their growth in host organisms.
\end{abstract}

\section{Introduction}

Pathogenic mycobacteria are relatively slow growing in laboratory culture media. Therefore, differential identification cannot be achieved for 3-12 weeks by conventional methods. Identification is important because the sensitivities of mycobacteria to antibiotics depends on the species. This is particularly so with the Mycobacterium avium - intracellulare - scrofulaceum (MAIS) group and the M. tuberculosis (tubercle) complex which can cause similar infections but show little similarity in their susceptibilities to antibiotics. Infections with these two groups of mycobacteria have increased in recent years, particularly in patients with AIDS. Infections with other less common mycobacterial pathogens are also important. These infections should be treated as quickly as possible, but there are few rapid methods to identify the causative organisms. Methods that identify mycobacteria by nucleic acid amplification and hybridisation are rapid and sensitive, but they usually require separate processing for each species, with unique probe sequences for each organism to be identified.

However, there are other biochemical methods for the identification of mycobacteria that have not been fully developed. These are based on the principle that mycobacteria produce a number of unique compounds. Whereas some compounds are unique to particular species, others, like the mycobactins which are involved in iron acquisition, have common structures but differ in the components of their side groups. ${ }^{1}$ Most species produce up to four molecular variants of mycobactins which differ in the position and length of the five alkyl chain residues. ${ }^{2}$ These molecules can be distinguished by extraction with alcohol followed by thin layer chromatography (TLC). ${ }^{1}$ The ability to identify mycobacteria by their mycobactin TLC sep-

Received 4 Oct. 1991 ; accepted 7 Jan. 1992. aration patterns was used in the chemotaxonomic identification of mycobacteria, ${ }^{3-7}$ but the methods required too many organisms to be of use in clinical identification. The aim of this study was to increase the sensitivity of this approach and thus provide a simple method that could be used routinely for the identification of mycobacteria.

\section{Materials and methods}

\section{Bacterial strains}

The following strains of mycobacteria were used: $M$. avium NCTC $8559, M$. intracellulare NCTC 10425

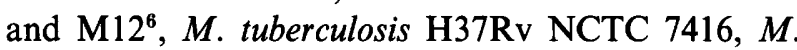
bovis NCTC $10772, M$. bovis var BCG NCTC 5692, $M$. scrofulaceum NCTC 10803, $M$. smegmatis NCIB $8548, M$. vaccae NCTC $10916, M$. fortuitum NCTC 10394 and $10395, M$. thermoresistible NCTC 10409, and $M$. neoaurum NCTC 10818.

\section{Media and growth conditions}

All strains were maintained on Lowenstein-Jensen medium after growth at $37^{\circ} \mathrm{C}$. For the expression of mycobactins, the organisms were grown on slopes of the following low-iron defined medium containing (/L): glucose $10 \mathrm{~g}$, glycerol $5 \mathrm{ml}$, asparagine $5 \mathrm{~g}$, $1.66 \mathrm{mM} \quad \mathrm{MgSO}_{4}, \quad 1.8 \mu \mathrm{M} \mathrm{MnSO}_{4}, \quad 7 \mu \mathrm{M} \mathrm{ZnSO}_{4}$, $0.18 \mu \mathrm{M} \mathrm{FeSO}_{4}$ and purified agar $1.5 \mathrm{~g}$. Where appropriate, $0.1 \mathrm{~mm}$ desferrioxamine mesylate (Sigma), $1 \mathrm{~mm} 2,2$ 'dipyridyl (Sigma) or $\beta$-cyclodextrin $0.5 \%$ $\mathrm{w} / \mathrm{v}$ were added to the cooled agar before it set.

\section{Extraction and labelling of the mycobactin}

Bacterial colonies were picked with a sterile plastic loop and placed in an Eppendorf centrifuge tube containing $0.5 \mathrm{ml}$ of either methanol or ethanol. The 
stem of the loop was removed and the loop plus alcohol was mixed for $15 \mathrm{~min}$ with a blood tube rotator. The tube was then centrifuged for $5 \mathrm{~min}$ at $13500 \mathrm{rpm}$ and the supernate was transferred to a new tube. The mycobactin was labelled with ${ }^{55} \mathrm{FeCl}$ (Code IES 3, Amersham International) $0.02 \mu \mathrm{Ci}$ in $20 \mu \mathrm{l}$ of sterile $20 \mu \mathrm{M} \mathrm{HCl}$ for $5 \mathrm{~min}$ on the tube rotator. The labelled mycobactin was extracted with $1 \mathrm{ml}$ of chloroform by gentle mixing for $3 \mathrm{~min}$. Distilled water $(100 \mu \mathrm{l})$ was added and the tubes centrifuged for $10 \mathrm{~s}$ at $13500 \mathrm{rpm}$. The methanol was discarded and the chloroform was removed by heating at $80^{\circ} \mathrm{C}$.

\section{Thin layer chromatography}

The radiolabelled sample was resuspended in $20 \mu 1$ of methanol and spotted on to a $10 \times 10 \mathrm{~cm}$ silica gel 60 high performance thin layer chromatography (HPTLC) plate with concentrating zone (Merck, Germany), or a $20 \times 20 \mathrm{~cm}$ silica gel TLC plate without concentrating zone (Merck). The samples were separated with petroleum spirit:butanol:ethyl acetate $2: 3: 3 \mathrm{v}: \mathrm{v}: \mathrm{v}$. At the completion of a run, the solvent front was marked with a spot of ${ }^{55} \mathrm{FeCl}$, and the plates were scanned with an AMBIS MK2, (AMBIS, USA), radio-analytic system ${ }^{8,9}$ for up to $18 \mathrm{~h}$ for the presence of radioactive spots.

\section{Results}

\section{Detection of fast-growing mycobacteria}

To test the feasibility of radiolabelling mycobactins to identify mycobacteria, three fast-growing mycobacterial strains, $M$. smegmatis, $M$. fortuitum NCTC 10394 and $M$. neoaurum, were grown on slopes of the defined medium at $37^{\circ} \mathrm{C}$ for 5 days. Sets of one, two, five or 10 colonies were picked. The cells were extracted with ethanol, labelled with ${ }^{55} \mathrm{FeCl} 0.02 \mu \mathrm{Ci}$ and separated on TLC plates without concentrating zones. Scans of these samples revealed that the method was only just sensitive enough to detect a single colony of $M$. neoaurum, but could easily detect single colonies of the other mycobacteria, which had formed bigger colonies. The overall resolution was poor, and as the sample size increased there were problems with lipids that bound iron and caused streaking of the plates.

\section{Detection of slow-growing mycobacteria}

M. tuberculosis H37Rv, M. tuberculosis H37Ra, $M$. avium, $M$. intracellulare NCTC $10425, M$. bovis NCTC 10772 and $M$. scrofulaceum were incubated at $37^{\circ} \mathrm{C}$ for 14 days on the defined medium and treated like the rapidly growing bacteria described above. The scans of these samples (fig. 1) showed that whilst diffuse spots could be detected for the tubercle bacilli at the expected Rf positions, ${ }^{7}$ no spots were detected for any members of the MAIS group. Therefore, these organisms were able to grow under conditions of lower iron concentration than the other species examined without showing the synthesis of mycobactin. M. bovis grew very poorly on this medium, presumably because of the presence of large amounts of glycerol, and failed to produce a detectable spot.

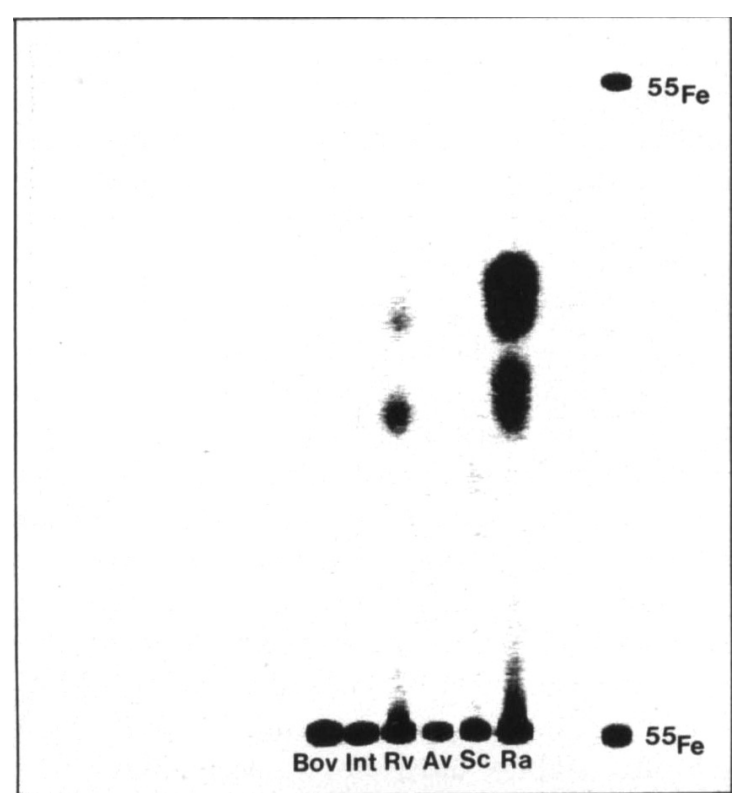

Fig. 1. Identification of slow-growing mycobacteria by radio-TLC. $M$. bovis (Bov), M. tuberculosis H37Rv (Rv), M. tuberculosis $\mathrm{H} 37 \mathrm{Ra}$ (Ra), M. avium (Av), M. scrofulaceum (Sc) and M. intracellulare (Int) were grown on iron-limiting medium for 14 days at $37^{\circ} \mathrm{C}$. Mycobactin extracts from two colonies of these cultures were labelled with ${ }^{55} \mathrm{Fe}$ and separated by TLC on silica gel 60 with petroleum spirit: butanol:ethyl acetate $2: 3: 3 \mathrm{v}: \mathrm{v}: \mathrm{v}$. The migration distance of the solvent is indicated with ${ }^{55} \mathrm{Fe}$ spots.

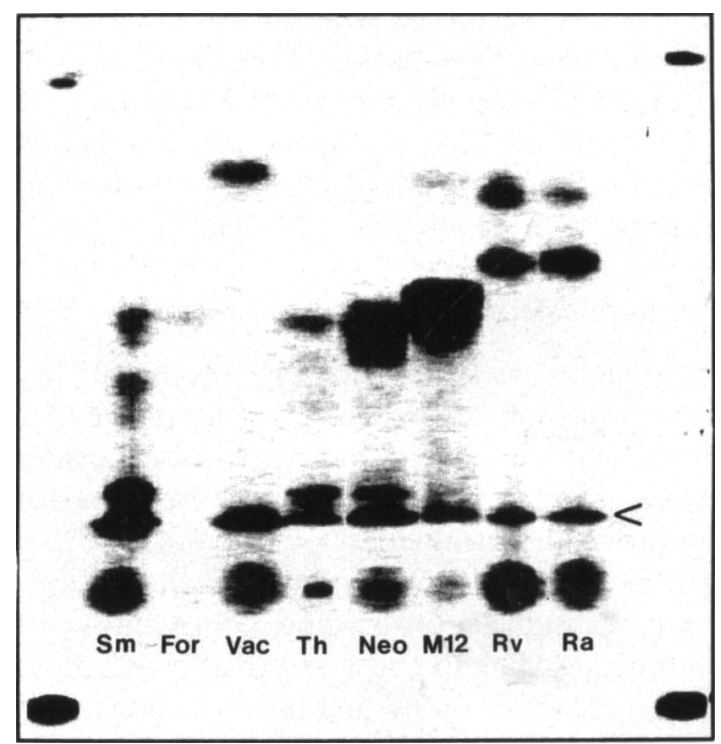

Fig. 2. Improved detection method. $M$. smegmatis $(\mathrm{Sm}), M$. fortuitum (For), $M$ vaccae (Vac), $M$. thermoresistible (Th) and $M$. neoaurum (Neo) were grown overnight, and $M$. intracellulare strain M12 (M12), M. tuberculosis H37Rv (Rv) and $M$. tuberculosis $\mathrm{H} 37 \mathrm{Ra}(\mathrm{Ra})$ were grown for 4 days at $37^{\circ} \mathrm{C}$. M. vaccae was grown in the presence of $0.1 \mathrm{~mm}$ desferrioxamine mesylate. Mycobactin extracts from a single colony of these cultures were labelled with ${ }^{55} \mathrm{Fe}$ and separated by TLC on a silica gel 60 plate with concentrating zone with petroleum spirit:butanol:ethyl acetate $2: 3: 3 \mathrm{v}: \mathrm{v}: \mathrm{v}$. The base of the plate and the solvent front are indicated with ${ }^{55} \mathrm{Fe}$ spots; $<$ indicates the end of the concentrating zone. 


\section{Improved detection method}

The method was made more sensitive by using radioactive iron with a high specific activity (Cat IES 3, Amersham International), which enabled shorter growth times for the bacteria. Thus, the fast-growing organisms could be detected from overnight growth and the slow growing organisms could be detected after incubation for 4 days (fig. 2). The TLC plates were replaced with HPTLC plates which improved the resolution and shortened the separation times, but this increased the cost of the system. The presence of interfering waxes was overcome by an initial extraction with methanol in place of ethanol (fig. 2).

Nearly all of the organisms produced bands with the expected Rf values. ${ }^{3,6,7}$ The exception was $M$. intracellulare strain $\mathrm{M} 12$ which seemed to have adapted to produce a previously uncharacterised mycobactin pattern. This organism showed its original culture and biochemical properties and produced chloroform-soluble exochelins as expected from $M$. intracellulare, and thus was not considered to be a contaminant. Changes have been observed in the mycobactin profiles of $M$. paratuberculosis NADC 18 and a strain of $M$. scrofulaceum. ${ }^{2}$ These changes, which are stable, have never been observed for mycobacteria other than those belonging to the MAIS group, and have been identified only in MAIS group organisms that have been grown for many subcultures in iron-deficient media. Thus, the expected profile for a freshly isolated strain would be that of a spot with an $\mathrm{Rf}$ of 0.89 with the solvent system as described.

By slightly modifying the solvent system to give ratios of petroleum spirit:ethyl acetate:butanol of 20:25:30 v:v:v, the separation patterns remained unchanged but differentiation of the single strain of $M$. bovis var BCG tested from tubercle bacilli was possible (fig. 3). The disadvantage of this method is that the Rf values obtained for all of the mycobactins tested differed from those already published.

\section{Mycobactin detection of MAIS organisms}

Mycobactin was occasionally produced by the MAIS group of organisms grown on the defined medium, and sometimes when grown on Lowenstein -Jensen medium, but the results were inconsistent. To improve the production of mycobactin, the medium was made more iron-limiting by the inclusion of the non-utilisable iron-binding compounds desferrioxamine mesylate $(0.1 \mathrm{~mm}$ or $1 \mathrm{~mm})$ or $2,2^{\prime}$ dipyridyl $(0.1 \mathrm{~mm}$ or $1 \mathrm{~mm})$, or by adding $\beta$-cyclodextrin. The cells grew poorly in the presence of 2,2'dipyridyl, taking $c$. twice as long to grow as the iron-sufficient controls. The cells grown in the presence of $0.1 \mathrm{~mm}$ desferrioxamine mesylate or $\beta$-cyclodextrin grew to form usable microcolonies within 4-6 days. The cells grown in these iron-limiting conditions consistently produced detectable amounts of mycobactins from single colonies (fig. 3). The amount of mycobactin produced by each was measured with the AMBIS

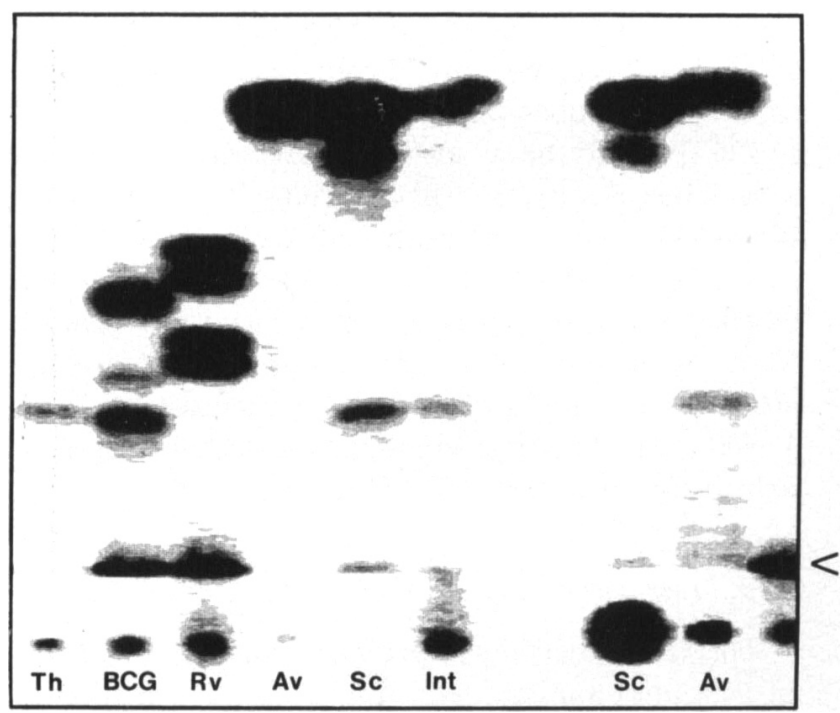

Fig. 3. Mycobactin production by MAIS group organisms. $M$. thermoresistible (Th) was grown overnight, and $M$. intracellulare (Int), M. avium (AV), M. scrofulaceum (Sc), M. tuberculosis H37Rv (Rv) and $M$. bovis var BCG (BCG) were grown for 4 days at $37^{\circ} \mathrm{C}$ on iron-limiting medium containing $0.1 \mathrm{mM}$ desferrioxamine mesylate. The $M$. avium (Av) and $M$. scrofulaceum (SC) samples were from cells grown on iron-limiting medium containing $\beta$-cyclodextrin $0.5 \% \mathrm{w} / \mathrm{v}$. Mycobactin extracts from a single colony of these cultures were labelled with ${ }^{55} \mathrm{Fe}$ and separated by TLC on a silica gel 60 plate with concentrating zone with petroleum spirit: butanol : ethyl acetate $20: 25: 30 \mathrm{v}: \mathrm{v}: \mathrm{v} ;<$ indicates the end of the concentrating zone.

scanner as cpm. From this it was calculated that the cells grown in the presence of $\beta$-cyclodextrin yielded $31-64 \%$ of the mycobactin produced by growth in the presence of desferrioxamine mesylate. In all cases the decreased growth of these organisms was overcome by the increase in sensitivity resulting from the increase in mycobactin production. The addition of desferrioxamine mesylate in amounts greater than $0.1 \mathrm{~mm}$ had no additional effect on the amounts of mycobactin produced by these organisms, but the growth of the bacteria was considerably reduced, and was completely inhibited, by a concentration of $5 \mathrm{~mm}$.

The early experiments with desferrioxamine and $\beta$ cyclodextrin were done with $M$. vaccae as a faster growing model organism for the MAIS group. $M$. vaccae is usually regarded as lacking the ability to produce mycobactin, although one mycobactin-positive strain has been reported. ${ }^{3}$ In agreement with these workers we were unable to demonstrate convincingly that mycobactin was produced by the type strain when grown on the iron-limiting medium alone. However, we found that if it was grown in the presence of desferrioxamine or $\beta$-cyclodextrin at $0.1 \mathrm{~mm}$, a band with an $\mathrm{Rf}$ similar to that reported previously for strain NCTC 10916, which produced trace amounts, was obtained (fig. 2). This suggests that mycobactin synthesis by $M$. vaccae might be increased or induced under some conditions of iron limitation.

\section{Sensitivity}

To calculate the sensitivity of the method, bacterial numbers were calculated by direct microscopical 
counting in tests with $M$. smegmatis. A calibration curve was constructed of cell numbers against radioactive cpm detected by the scanner. Desferrioxamine mesylate was included in the agar, and radioactive iron with a high specific activity was used. The sensitivity of the method was calculated to be $2.87 \times 10^{3}$ bacteria. In theory this should be adequate for the direct detection of bacteria in sputum as most sputum samples contain more than $10^{3}$ cells $/ \mathrm{ml}$.

\section{Discussion}

We have refined the identification method originally proposed by Snow and White ${ }^{1}$ and developed for taxonomic purposes ${ }^{3}$ for use in the routine identification of mycobacteria, based on the published Rf values of a large number of representative species. ${ }^{2-7}$ The method requires the calculation of the $R f$ values of the mycobactins, which is not difficult but could be eliminated by comparing the patterns obtained with a set of known mycobactins run on a track alongside the samples, as size markers are used in SDS-PAGE of proteins. Rapidly growing mycobacteria can be identified within $24 \mathrm{~h}$, and slow growing ones within 5 days. Most mycobacterial species can be identified within a few hours if sufficient organisms are available. A scanner was used in these experiments to detect the radioactivity but $\mathrm{X}$-ray film could be used if a scanner was not available although the exposure times would be longer.

The limitation of this approach is that mycobacteria that do not produce mycobactins cannot be identified. Three species do not produce mycobactins in substantial quantities- $M$. paratuberculosis, $M$. vaccae and $M$. leprae. The first two have the potential to produce mycobactins $\mathrm{s}^{3,6,10}$ and the small amounts produced normally can be increased by growing the organisms on iron-limiting medium containing desferrioxamine mesylate. However, $M$. paratuberculosis grows so slowly under iron-limited conditions (some strains take 18 months to grow) that the technique is probably impractical for this organism. Although $M$. leprae has not been shown to produce mycobactin, it

\section{References}

1. Snow GA, White AJ. Chemical and biological properties of mycobactins isolated from various mycobacteria. Biochem $J$ 1969; 115: 1031-1045.

2. Barclay R, Ewing DF, Ratledge C. Isolation, identification and structural analysis of the mycobactins of Mycobacterium avium, Mycobacterium intracellulare, Mycobacterium scrofulaceum, and Mycobacterium paratuberculosis. $J$ Bacteriol 1985; 164: 896-903.

3. Hall RM, Ratledge C. Mycobactins as chemotaxonomic characters for some rapidly growing mycobacteria. $J$ Gen Microbiol 1984; 130: 1883-1892.

4. Hall RM, Ratledge C. Equivalence of mycobactins from Mycobacterium senegalense, Mycobacterium farcinogenes has not as yet been tested by this highly sensitive method.

The results of the study have highlighted two atypical properties of mycobactin production, and thus iron-acquisition, by the MAIS group. The first is that they grow in conditions that would be ironlimiting to the other Mycobacterium spp. tested without producing mycobactin. This indicates that the MAIS group have a lower requirement for iron than the other mycobacteria, or that they use their exochelin-mediated iron uptake systems more effectively. The lower requirement for iron by the MAIS group would explain whey $M$. avium has been observed $^{11}$ to grow transiently under iron-sufficient conditions in man. Transient changes clearly create problems in using this identification method directly on clinical specimens, as MAIS strains might not always synthesise detectable mycobactins. Thus, for the guaranteed detection of the MAIS group the cell would need to be grown on an iron-limiting medium, such as that used in this study.

The second unusual property of the MAIS group is the change in the profiles obtained after many subcultures under low iron conditions. The properties of these organisms are almost identical to those of the original isolates. The differences are in the mycobactin profiles, and in a 2-3-fold shortening of the growth time of the altered cells. This indicates that mutants of the MAIS group are being selected during growth in the iron-limiting medium, and could explain the discrepancies observed previously for mycobactin " $\mathrm{J}$ " from $M$. paratuberculosis NADC $18,{ }^{2}$ with which two different mycobactin profiles were obtained.

The most common Mycobacteria found in clinical situations are $M$. tuberculosis and the MAIS group of organisms, but there is also the risk of serious infection by organisms such as $M$. fortuitum and $M$. chelonae and others from hospital water supplies, operating theatres and haemodyalis units. Our main concern has been the identification of mycobacteria that cause disease, but the monitoring of mycobacteria in the environment is also important. The use of radiolabelled mycobactin provides a simple system for the identification of mycobacteria under all of these circumstances. and Mycobacterium fortuitum. J Gen Microbiol 1985; 131 : 1691-1696.

5. Hall RM, Ratledge C. Mycobactins in the classification and identification of armadillo-derived mycobacteria. FEMS Microbiol Lett 1985; 28: 243-247.

6. Barclay R, Ratledge C. Iron-binding compounds of Mycobacterium avium, $M$. intracellulare, $M$. scrofulaceum, and mycobactin-dependent $M$. paratuberculosis and $M$. avium. $J$ Bacteriol 1983; 153: 1138-1146.

7. Barclay R, Ratledge C. Mycobactins and exochelins of $M y c o-$ bacterium tuberculosis, $M$. bovis, $M$. africanum and other related species. J Gen Microbiol 1988; 134: 771-776.

8. Smith I. The AMBIS beta scanning system. Bioassays $1985 ; 3$ : 225-229.

9. Smith I, Furst V. Comparative analysis of radiochroma- 
tography of gels. Qualitative and quantitative methods with the AMBIS system. J Planar Chrom 1989; 2: 233-237. 10. Merkal RS, McCullough WG, Takayama K. Mycobactins, the state of the art. Bull Inst Pasteur 1981; 79: 251-259.
11. Sritharan $M$, Ratledge $C$. Iron-regulated envelope proteins of mycobacteria grown in vitro and their occurrence in Mycobacterium avium and Mycobacterium leprae grown in vivo. Biol Met 1990; 2: 203-208. 